\title{
Mutation of the Xylanase regulator 1 causes a glucose blind hydrolase expressing phenotype in industrially used Trichoderma strains
}

\author{
Christian Derntl ${ }^{1}$, Loreta Gudynaite-Savitch ${ }^{2}$, Sophie Calixte ${ }^{2}$, Theresa White ${ }^{2}$, Robert L Mach ${ }^{1}$ \\ and Astrid R Mach-Aigner ${ }^{1 *}$
}

\begin{abstract}
Background: Trichoderma reesei is an organism involved in degradation of (hemi)cellulosic biomass. Consequently, the corresponding enzymes are commonly used in different types of industries, and recently gained considerable importance for production of second-generation biofuel. Many industrial T. reesei strains currently in use are derived from strain Rut-C30, in which cellulase and hemicellulase expression is released from carbon catabolite repression. Nevertheless, inducing substances are still necessary for a satisfactory amount of protein formation.

Results: Here, we report on a T. reesei strain, which exhibits a very high level of xylanase expression regardless if inducing substances (e.g. D-xylose, xylobiose) are used. We found that a single point mutation in the gene encoding the Xylanase regulator 1 (Xyr1) is responsible for this strong deregulation of endo-xylanase expression and, moreover, a highly elevated basal level of cellulase expression. This point mutation is localized in a domain that is common in binuclear zinc cluster transcription factors. Only the use of sophorose as inducer still leads to a slight induction of cellulase expression. Under all tested conditions, the formation of cbh1 and cbh2 transcript level strictly follows the transcript levels of $x y r 1$. The correlation of $x y r 1$ transcript levels and cbh $1 / c b h 2$ transcript levels and also their inducibility via sophorose is not restricted to this strain, but occurs in all ancestor strains up to the wild-type QM6a.
\end{abstract}

Conclusions: Engineering a key transcription factor of a target regulon seems to be a promising strategy in order to increase enzymes yields independent of the used substrate or inducer. The regulatory domain where the described mutation is located is certainly an interesting research target for all organisms that also depend so far on certain inducing conditions.

Keywords: Trichoderma reesei, Hypocrea jecorina, Cellulases and hemicellulases, Inducer-independent enzyme production, Biofuel, Xylanase regulator 1 (Xyr1), Glucose response domain

\section{Background}

Trichoderma reesei (telomorph, Hyprocrea jecorina) [1] is a filamentous ascomycete thriving as a saprophyte on dead plant material. It degrades cellulose and hemicelluloses by secreting a wide array of cellulases and hemicellulases. A genome-wide analysis revealed 10 celluloytic and 16 xylanolytic enzyme-encoding genes in $T$. reesei [2]. The most abundantly secreted and industrially interesting

\footnotetext{
* Correspondence: astrid.mach-aigner@tuwien.ac.at

'Department for Biotechnology and Microbiology, Institute of Chemical Engineering, Vienna University of Technology, Gumpendorfer Str. 1a, A-1060, Wien, Austria

Full list of author information is available at the end of the article
}

enzymes are the two main cellobiohydrolases, CBHI and CBHII (EC 3.2.1.91) [3], and two major specific endo-ß-1,4-xylanases, XYNI and XYNII (EC.3.2.1.8) [4]. We will use the term major, industrially relevant hemicellulases and cellulases (MIHCs) throughout this publication for these two cellulases and two hemicellulases.

The MIHCs work together with further enzymes to degrade cellulose and xylan. This results in the formation of soluble oligo- and monosaccharides, such as cellobiose, D-glucose, xylobiose, and D-xylose. In addition, sophorose is a product of the transglycosylation activity of some of these enzymes [5]. All of these molecules were reported to

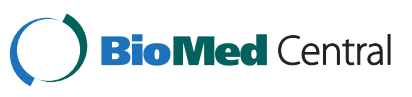


have influence on the expression of MIHCs in T. reesei. The presence of D-glucose causes carbon catabolite repression (CCR), which results in the secretion of low quantities of MIHCs; expression of XYNI is even completely shut off. Sophorose is the most potent inducer for the expression of $\mathrm{CBHI}$ and $\mathrm{CBHII}$. It also triggers the expression of XYNII (reviewed by [6]). D-xylose modulates XYNI and XYNII expression in a concentration-dependent manner. Strongest induction occurs by using low concentrations $(0.5 \mathrm{mM})$, whereas high concentrations lead to a repressing effect on xylanase expression [7].

Despite the different patter of inducibility of the expression of MIHCs, it generally depends on the presence of the main transactivator of hydrolases Xyr1 (Xylanase regulator 1). Xyr1 has a Gal4-like $\mathrm{Zn}_{2} \mathrm{Cys}_{6}$ binuclear cluster domain, which is involved in DNA-binding. A xyr1 deletion mutant does not produce any MIHCs at the level of either transcription or of protein formation [8,9]. It has been reported that using D-xylose or xylobiose could not induce the expression of xyr1 itself, even if these saccharides are potent inducers of XYNI and XYNII expression mediated via Xyr1. However, analogous to its target genes, Xyr1 expression is regulated by CCR mediated by Cre 1 [10]. Cre1 is a wide-domain regulator that binds under repressing conductions (high concentrations of easily utilisable monosaccharides such as D-glucose or D-xylose) to its binding site in the promoter of e.g. $x y r 1$ or $x y n 1$ resulting in a down-regulation or a complete shut-off of transcription, respectively [11-13].

It is evident that a release from CCR is a useful prerequisite for industrial exploitation of $T$. reesei for the production of MIHCs. Therefore, a prominent Cre1-deficient mutant strain of $T$. reesei, Rut-C30, which was described as a high yielding cellulase mutant [14], has been used as the progenitor strain for many recent industrial strains. However, industrially satisfying production of MIHCs by Rut-C30 and its industrially used offspring still depends on potent induction. Using certain inducing compounds or a particular media composition is the common way to achieve this induction, but both add cost which may lead to a higher price for the resulting enzyme products. In particular, the economical, ecological and socio-economical success of products such as second-generation biofuel strongly depend on their cheap availability of MIHCs as well as a production process using non-food biomass as starting material.

In this study we report on an industrially used T. reesei strain that produces high amounts of MIHCs independent of the presence of a certain inducer. Moreover, this strain shows a glucose-blind phenotype when it comes to expression of MIHCs. Consequently, we analysed this strain at the transcriptional level in order to identify the molecular mechanisms behind this phenotype. Interestingly, we found aside to other mutations a single point mutation in Xyr1 and investigated to which extent this is responsible for the outstanding phenotype of that strain.

\section{Results}

A two-step mutant derived from $T$. reesei Rut-C30 yields elevated and aberrant xylanase expression due to a single point mutation in $x y r 1$

The industrial strain Iogen-M4 (derived from Rut-C30 by spontaneous mutation) was used as parental strain for UV mutagenesis. During the subsequent screening process the mutant Iogen-M8 was selected due to its elevated xylanase activity. Cultivation in a bioreactor revealed a generally elevated protein secretion rate and confirmed an increase of xylanase expression compared to its parental strain. High levels of XYNI and XYNII could even be found in the supernatant when cellulase inducing conditions were applied. An additional figure presenting ELISA data shows this more in detail (see Additional file 1). In order to identify a possible genetic cause for this behaviour, the genomes of Iogen-M4 and Iogen-M8 were sequenced. The two strains differ in 17 point mutations within ORFs giving rise to mutations in known or predicted proteins. One of which is a point mutation within xyr 1 manifesting in the expression of an altered Xyr1 having alanine replaced by valine at position 824 (A824V). The complete sequences of the wild-type and mutated $x y r 1$ and Xyr 1 is given in an additional file (see Additional file 2). This point mutation was introduced into Iogen-M4 resulting in the strain Iogen-M4X. This strain leads to an identical expression of cellulases and hemicellulases behaviour like Iogen-M8. Vice versa, re-establishing the wild-type $x y r 1$ in Iogen-M8 resulted in the strain Iogen-M8X exhibiting xylanase expression levels comparable to the parental strain Iogen-M4. Figure 1 gives an overview of these strains, their pedigree, and their xylanolytic properties when grown on xylan plates.

\section{Strong deregulation of expression of MIHCs can be observed in T. reesei logen-M8}

The outstanding properties of Iogen-M8 with regard to xylanolytic activity were investigated on the level of transcription to identify changes in regulatory mechanisms of expression of MIHCs compared to Rut-C30, its first ancestor with a Cre1-negative background. Therefore, both strains were pre-grown in Mandels-Andreotti (MA) medium containing solely glycerol as carbon source. These mycelia were replaced into MA medium containing either $50 \mathrm{mM}$ D-glucose, $0.5 \mathrm{mM}$ D-xylose, $66 \mathrm{mM}$ D-xylose, or $1.5 \mathrm{mM}$ sophorose as carbon sources or inducers $[7,15,16]$. An additional culture was incubated in MA medium without carbon source as reference. Samples were taken directly from the pre-culture (before the replacement) and after three and six hours of incubation. RNA of these samples was extracted and used as template for RT-qPCR analysis. 


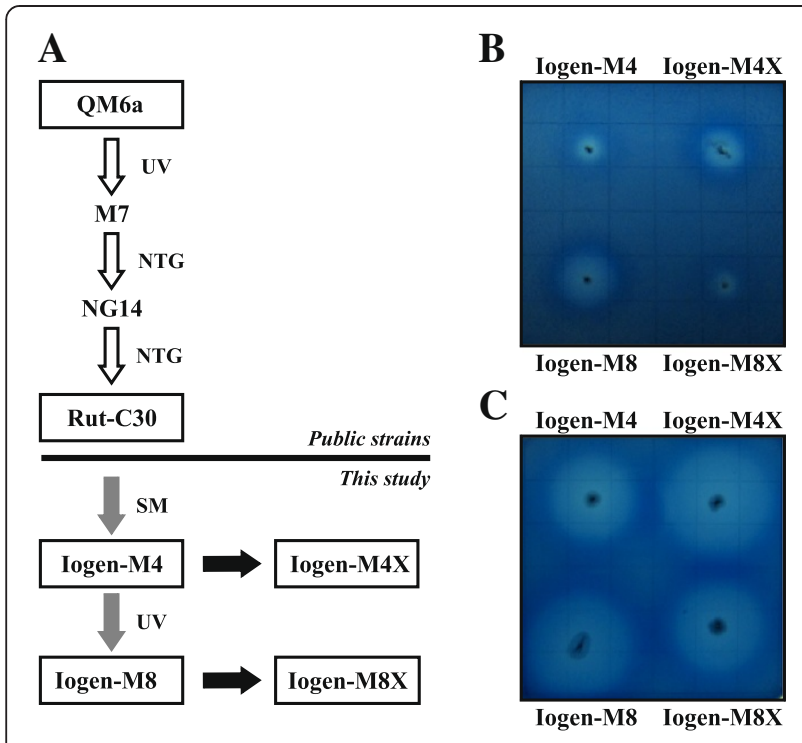

Figure 1 Pedigree and xylanolytic properties of $T$. reesei strains used in this study. (A) Schematic drawing of the pedigree of strains of which investigated ones are shown in boxes. Next to arrows the method of strain generation is indicated: UV-mutagenesis (UV), nitrosoguanidine (NTG), spontaneous mutation (SM). White arrows indicate strain generation before this study, grey arrows indicate strain generation during this study, black arrows indicate strains generated by targeted gene replacement during this study. Xylanolytic properties of strains generated during this study are represented by growth on plates containing $0.2 \%(\mathrm{w} / \mathrm{v})$ azo-xylan as carbon source at $30^{\circ} \mathrm{C}$ for $24 \mathrm{~h}(\mathrm{~B})$ and for $72 \mathrm{~h}$ followed by a $2 \mathrm{~h}$ incubation at $50^{\circ} \mathrm{C}(\mathbf{C})$.

Transcript levels of $x y n 1$ and $x y n 2$ of Iogen-M8 could be detected at a constantly high level, regardless if any, or which, carbon source or inducer was used (Figure 2A, B). This finding correlates with the elevated and deregulated xylanolytic enzyme formation observed previously during the UV mutant screening procedure. Generally, Iogen-M8 shows considerably higher transcript levels of all tested genes under all conditions compared to Rut-C30 (compare Figure 2A-E to Figure 2F-J); e.g. transcript levels of $x y n 1$ and $x y n 2$ are 100 - to 10,000 -fold increased compared to those in Rut-C30 (compare Figure 2A to Figure 2F and Figure $2 \mathrm{~B}$ to Figure $2 \mathrm{G}$, respectively). In contrast, in Rut-C30, other than a general, slight deregulation, the genes $x y n 1$ and $x y n 2$ are both inducible by $\mathrm{D}$-xylose (Figure 2F, G).

In addition, the transcript levels of the main cellobiohydrolase-encoding genes, $c b h 1$ and $c b h 2$, showed a striking increase (up to 10,000-fold) in Iogen-M8 compared to Rut-C30 (compare Figure 2C, D to Figure 2H, I). These genes remain subject to induction in Iogen-M8 as a comparison of their transcript levels derived from incubation without carbon source to those with sophorose reveals (Figure 2C, D), even if the extent of inducibility is less pronounced than in Rut-C30 (Figure 2H, I). Notably, in both strains xyr1 transcription is inducible by sophorose (Figure 2E, J), and the patterns of transcript levels of cbh1 and cbh2 reflect those of xyr1 on all carbon sources or inducers tested (Figure 2C-E and Figure $2 \mathrm{H}-\mathrm{J}$ ).

In summary, Iogen-M8 has general highly elevated transcript levels of all genes under all investigated conditions compared to Rut-C30. Consequently, we conclude Iogen-M8 exhibits a strong deregulation in expression of MIHCs.

\section{Deregulation of MIHCs expression only partly occurs in the parental strain of logen-M8}

The comparison of transcript levels of all MIHCs-encoding genes revealed strong differences between Iogen-M8 and Rut-C30. As two mutation/selection steps gap these two strains, we examined the intermediate strain Iogen-M4 in order to see what the first step and what the second step contribute to the observed phenotype of Iogen-M8. Therefore, we performed a carbon source replacement experiment applying the same conditions as before and analyzed the transcript levels. Like Iogen-M8, Iogen-M4 also exhibits higher transcript levels of all investigated genes under all conditions tested compared to Rut-C30 (compare Figure $3 \mathrm{~A}-\mathrm{B}$ to Figure $2 \mathrm{~F}-\mathrm{J})$. However, the rise of transcript levels is less pronounced in Iogen-M4 than in Iogen-M8 (compare Figure $3 \mathrm{~A}-\mathrm{E}$ to Figure 2A-E). $\mathrm{D}$-xylose at low and high concentrations induces $x y n 1$ and $x y n 2$ (compared to no carbon source) in Iogen-M4 (Figure 3A, B). The same is true for Rut-C30 (Figure 2F, G), while in Iogen-M8 the high $x y n 1$ and $x y n 2$ transcript levels remain unaffected regardless if D-xylose is applied or not (Figure 2A, B). Sophorose induces cbh1 and cbh2 gene expression in Iogen-M4 (Figure $3 \mathrm{C}-\mathrm{E}$ ) as already observed before for Rut-C30 (Figure 2H-J) and, to a lesser extent, for Iogen-M8 (Figure 2C-E). In summary, we found elevated transcript levels of all MIHCs-encoding genes already in Iogen-M4 (compared to Rut-C30), but to less pronounced extent than in Iogen-M8. Moreover, the deregulation of xylanase gene expression observed in Iogen-M8 cannot be seen in Iogen-M4.

\section{A824V transition in Xyr1 causes strong deregulation of xylanase expression and elevated basal cellulase expression}

As previously stated, 17 point mutations in ORFs were identified in Iogen-M8 in comparison to Iogen-M4. We found that the A824V transition in Xyr1 leads to the elevated expression of xylanases in Iogen-M8. In order to test whether this mutation is solely responsible for all observed differences in transcript levels of Iogen-M4 and Iogen-M8, we performed transcript analysis of Iogen-M4X. This strain is isogenic to Iogen-M4 with the only exception of expressing the altered A824V (i.e. the Iogen-M8 like) Xyr1 (Figure 1A). Transcript levels of all investigated genes 

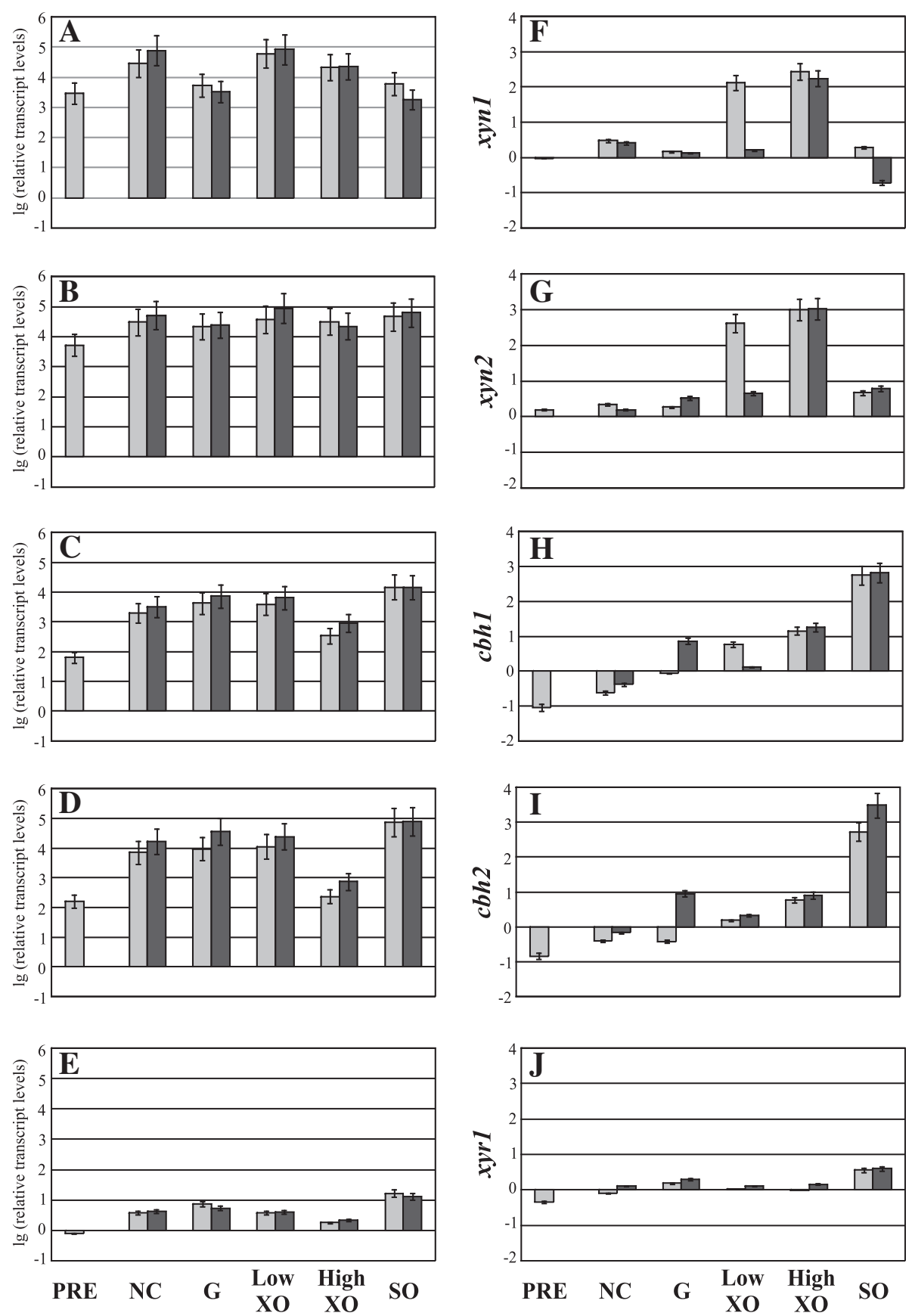

Figure 2 Analysis of transcript levels of MIHCs-encoding genes and xyr1 in T. reesei RutC30 and logen-M8. The xylanase overexpression strain logen-M8 (A-E) and its ancestor strain Rut-C30 (F-J) were precultured on glycerol and thereafter transferred to MA media without carbon source $(\mathrm{NC})$ or containing $50 \mathrm{mM}(\mathrm{W} / \mathrm{v})$ glucose $(\mathbf{G})$ or D-xylose $(\mathrm{XO})$ at the indicated concentration, or $1.5 \mathrm{mM}$ sophorose (SO) as an inducer. Samples were taken directly before transfer of mycelium (PRE), after $3 \mathrm{~h}$ of incubation (light grey bars), and after $6 \mathrm{~h}$ of incubation (dark grey bars). Transcription analyses of indicated genes from both strains were performed via qPCR using sarl and act transcript levels for normalization. Results are given as relative transcript ratios in logarithmic scale $(\mathrm{lg})$. The values provided in the figures are means from three biological experiments. Error bars indicate standard deviations. Transcript levels always refer to the reference sample (wild-type QM6a, NC 3 h).

(xyn1, xyn2, cbh1, cbh2, xyr1) were nearly the same in Iogen-M4X and Iogen-M8 (compare Figure 2A-E to Figure 4A-E). Consequently, we conclude that the A824V transition in Xyr1 is responsible for the strong deregulation of xylanase expression and the additional rise in transcript levels of $x y n 1$ and $x y n 2$ observed in Iogen-M8 (higher than in Iogen-M4) as well as the high basal transcript levels of cbh1 and cbh2. 
Figure 3 Analysis of transcript levels of MIHCs-encoding genes and $x y r 1$ in $T$. reesei logen-M4. The parental strain of logen-M8, logen-M4, was precultured on glycerol and thereafter transferred to MA media without carbon source (NC) or containing $50 \mathrm{mM}(\mathrm{W} / \mathrm{v})$ glucose (G) or D-xylose (XO) at the indicated concentration, or $1.5 \mathrm{mM}$ sophorose (SO) as an inducer. Samples were taken directly before transfer of mycelium (PRE), after $3 \mathrm{~h}$ of incubation (light grey bars), and after $6 \mathrm{~h}$ of incubation (dark grey bars). Transcription analyses of indicated genes (A-E) were performed via qPCR using sar1 and act transcript levels for normalization. Results are given as relative transcript ratios in logarithmic scale $(\mathrm{lg})$. The values provided in the figures are means from three biological experiments. Error bars indicate standard deviations. Transcript levels always refer to the reference sample (wild-type QM6a, NC 3 h).

In accordance with all other strains we could observe that $x y r 1, c b h 1$, and $c b h 2$ are inducible by sophorose in Iogen-M4X (Figure 4C-E). As a consequence of this observation, we questioned if this is due to a principle regulatory mechanism also present in the wild-type QM6a and not restricted to the strains investigated in this study so far (i.e., a consequence of mutagenesis and selection).

\section{Gene expression of $c b h 1$ and $c b h 2$ strictly depends on the} level of $x y r 1$ transcription

Notably, transcript levels of xyr1 are induced on sophorose in all strains investigated in this study so far (Figure 2E, J, Figure 3E, and Figure 4E). Moreover, the patterns of transcript levels of $c b h 1$ and $c b h 2$ strictly reflect those of $x y r 1$ under all conditions tested (Figure 2C-E, Figure 2H-J, Figure 3C-E, and Figure 4C-E). As mentioned, all these strains are derivatives of Rut-C30, a mutant derived from the wild-type strain QM6a (Figure 1A). We questioned whether the correlation between xyr 1 transcript levels and those of cbh1 and cbh2 can already be observed in the wild-type strain and performed an analogous carbon source replacement experiment using QM6a.

Sophorose induces $x y r 1$ transcription also in the wildtype (Figure 5E). In concordance with the results obtained with the other strains, $c b h 1$ and $c b h 2$ transcript levels are also elevated in QM6a on sophorose and generally reflect the carbon source/inducer-dependent pattern of xyr1 transcript levels (Figure 5C-E). In summary, elevated levels of xyr 1 seem to correlate directly with up-regulation of $c b h 1$ and $c b h 2$ transcription in all investigated $T$. reesei strains (compare Figures 2, 3, 4 and 5C-E). In contrast, xylanase expression in all investigated strains including the wild-type strain does not follow changes in xyr 1 expression levels, i.e. Xyr1 transactivates the xylanase regulon in a concentration-independent manner (compare Figures 2, 3, 4 and 5A, B, E). 
Figure 4 Analysis of transcript levels of MIHCs-encoding genes and $x y r 1$ in $T$. reesei logen-M4X. logen-M4X, which is logen-M4 with the mutated Xyr1, was precultured on glycerol and thereafter transferred to MA media without carbon source $(\mathrm{NC})$ or containing $50 \mathrm{mM}(\mathrm{w} / \mathrm{v})$ glucose $(\mathbf{G})$ or D-xylose $(\mathrm{XO})$ at the indicated concentration, or $1.5 \mathrm{mM}$ sophorose (SO) as an inducer. Samples were taken directly before transfer of mycelium (PRE), after $3 \mathrm{~h}$ of incubation (light grey bars), and after $6 \mathrm{~h}$ of incubation (dark grey bars). Transcription analyses of indicated genes (A-E) were performed via QPCR using sar1 and act transcript levels for normalization. Results are given as relative transcript ratios in logarithmic scale $(\mathrm{lg})$. The values provided in the figures are means from three biological experiments. Error bars indicate standard deviations. Transcript levels always refer to the reference sample (wild-type QM6a, NC 3 h).

\section{In silico characterization of the $x y r 1$ sequence part that bears the A824V transition}

According to DELTA-BLAST (http://www.ncbi.nlm.nih. gov/), Xyr1 has a so-called fungal transcription factor regulatory middle homology region (FTFRMH region) (from L359 to L904, cd12148 with an E-value of $1.77 \mathrm{e}^{-11}$ ) located next to the Gal4-like DNA-binding domain (from R93 to Y126, cd00067 with an E-value of $2.81 \mathrm{e}^{-10}$ ). The A824V mutation of Xyr1 is located within the FTFRMH region. This FTFRMH region is present in the large family of fungal zinc cluster transcription factors that contain an N-terminal GAL4-like Cys6 zinc binuclear cluster DNA-binding domain [17]. The C-terminal domain of Cep3p, a subunit of the yeast centromere-binding factor 3, is similar to the FTFRMH region (E-value $1.22 \mathrm{e}^{-04}$ ). A 3D model is available for a great part of Cep3p based on X-ray diffraction [18]. An alignment of the FTFRMH regions of Xyr1, Cep3p, and the consensus sequence matches position A824 of Xyr1 with I463 of Cep3p (Constraint-based Multiple Alignment Tool, http://www.ncbi.nlm.nih.gov/) and is shown in an additional file [see Additional file 3]. I463 of Cep3p is located in the middle of an $\alpha$-helix, which reaches from M458 to I475. A graphic display is given in an additional file (see Additional file 4). Additionally, three different secondary structure predictions for the domain of Xyr1 locate A824 in the middle of an $\alpha$-helix (http://www. compbio.dundee.ac.uk/, BCL::Jufo9D at http://meilerlab. org/, http://bioinf.cs.ucl.ac.uk/psipred/). Consequently, we assume that the A824V mutation in Xyr1 possibly leads to a change in secondary structure.

\section{Discussion}

During this study we found that the expression of the two major cellulase genes $c b h 1$ and cbh 2 strictly follow xyr 1 transcript levels. Accordingly, Portnoy and co-workers reported that in a cellulase-overproducing strain, xyr1 transcript levels are elevated compared to common $T$. reesei strains (as QM9414) [19]. These findings suggest 


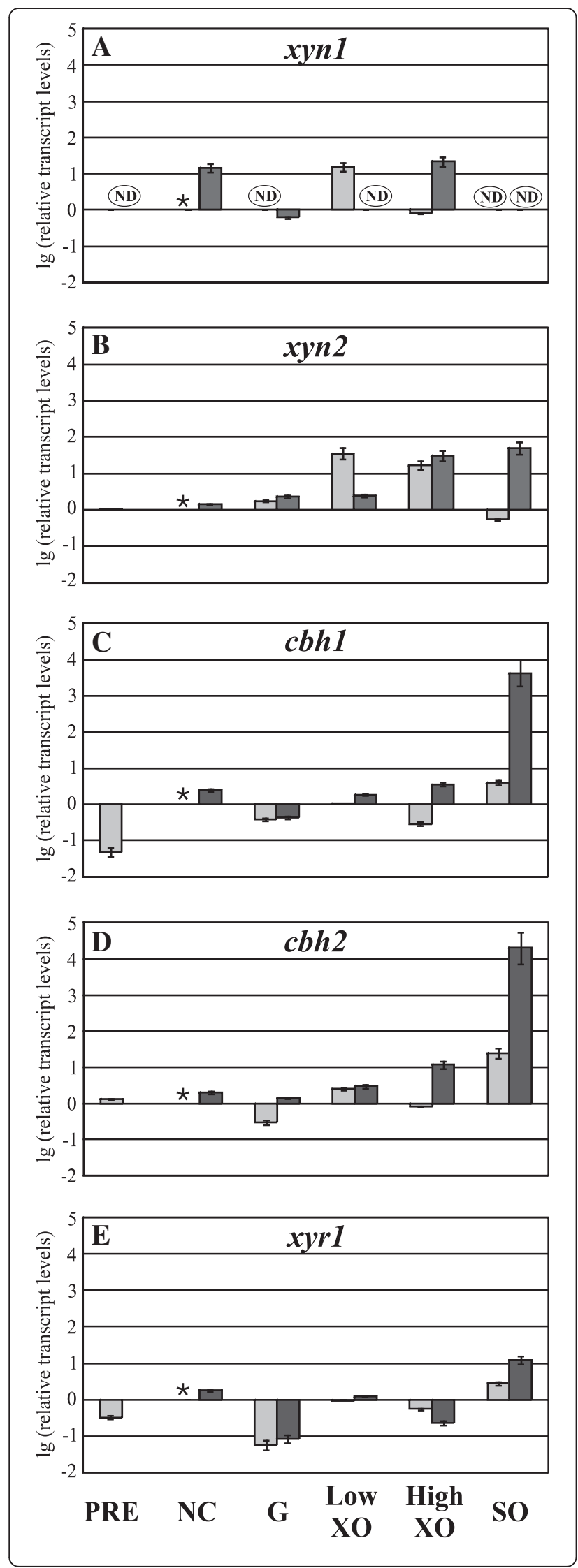

Figure 5 Analysis of transcript levels of MIHCs-encoding genes and $x y r 1$ in T. reesei QM6a. The wild-type QM6a was precultured on glycerol and thereafter transferred to MA media without carbon source (NC) or containing $50 \mathrm{mM}(\mathrm{w} / \mathrm{v})$ glucose $(\mathbf{G})$ or D-xylose (XO) at the indicated concentration, or $1.5 \mathrm{mM}$ sophorose (SO) as an inducer. Samples were taken directly before transfer of mycelium (PRE), after $3 \mathrm{~h}$ of incubation (light grey bars), and after $6 \mathrm{~h}$ of incubation (dark grey bars). Transcription analyses of indicated genes (A-E) were performed via qPCR using sar1 and act transcript levels for normalization. Results are given as relative transcript ratios in logarithmic scale $(\mathrm{lg})$. The values provided in the figures are means from three biological experiments. Error bars indicate standard deviations. Transcript levels always refer to the reference sample (wild-type QM6a, NC 3 h). * indicates the reference sample, ND means not detected.

that cellulase gene expression is highly dependent on the amount of Xyr1.

Otherwise, we found that the expression of the two major xylanases, $x y n 1$ and $x y n 2$, does not strictly follow xyr 1 transcript levels. Interestingly, in Aspergillus nidulans a constitutive expression of $x \ln R$ (the $x y r 1$ homolog [20]) under the gpdA promoter led to enhanced and continuously high $x \ln A / B$ transcript formation, while $x \ln D$ transcript diminished after $1 \mathrm{~h}$ and did not follow the $x \ln R$ transcript level pattern anymore [21]. Altogether, we assume that regulation of xylanase gene expression is not directly dependent on the amount of Xyr1 and seems to rely on additional mechanisms.

The different Xyr1 responsiveness of cellulases and xylanases was also observed in a T. reesei QM9414 strain constitutively expressing xyr1. There, the cellulolytic regulon of Xyr1 was positively affected, while the xylanolytic regulon was negatively affected [22]. Notably, this observations are supported by the number of in silico identified Xyr1-binding sites in respective promoter regions. The reported Xyr1-binding site, 5'-GGC(T/A) $)_{3}-3$ ' [23-26], occurs 14 times within $1 \mathrm{~kb}$ of the promoter region of $c b h 1$, whereas it occurs only 4 times within $1 \mathrm{~kb}$ of the promoter region of xyn1. Currently ongoing in vivo footprinting analysis of corresponding promoters revealed that 12 and 2 of these sites are differently contacted comparing inducing and repressing conditions, respectively (unpublished observations, Gorsche, R., Lichti, J., Mach, R.L., Mach-Aigner, A.R.). Supportively, we found during this study that sophorose, which has been known for decades as a potent cellulase inducer [15], positively influences $x y r 1$ expression. As stated before, expression of the two major cellulase genes cbh1 and cbh2 strictly follow xyr1 transcript levels. Taken together, we assume, that induction of $c b h 1$ and $c b h 2$ by sophorose is a direct result of elevated xyr1 transcription levels. The issues discussed so far could be observed in all of the $T$. reesei strains investigated in this study, including the wild-type QM6a. 
However, two outstanding phenomena could be observed in Iogen-M8: first, a strong deregulation of xylanase expression and second, a generally very high level of transcript formation of MIHCs. By investigating correspondingly manipulated strains, we found that a single point mutation in Xyr1 (A824V) is fully responsible for the deregulation of xylanase gene expression and the high basal level of $c b h 1$ and $c b h 2$ expression. A similar phenomenon was briefly described for the $\mathrm{X} \ln \mathrm{R}$ in $A$. niger. A V756F mutation resulted in constant xylanase activity even under repressing conditions [27]. Nonetheless, we currently cannot provide a mechanistic explanation, it is noteworthy that the A $824 \mathrm{~V}$ transition is located in a predicted $\alpha$-helix within a FTFRMH region. As alanine has the lowest helix propensity $(0 \mathrm{kcal} / \mathrm{mol})$, whereas valine has a higher value $(0.61 \mathrm{kcal} / \mathrm{mol})$ [28]. The mutation in Xyr1 may result in a structural change. Notably, the previously mentioned V756 in XlnR corresponds to V821 in Xyr1 located in the same predicted $\alpha$-helix as A824.

For Gal4 it was reported that glucose has a direct effect on its activity. The localisation of the glucose response domain in Gal4 was narrowed down to a central region [29], in which the FTFRMH region lies (E-value $\left.4.57 \mathrm{e}^{-50}\right)$. Albeit a functional similarity of both regions seems likely, we found that the phenotype of Iogen-M8 is not linked just to D-glucose. Consequently, we presume that the corresponding domain of Xyr1 is a more generally regulatory region.

\section{Conclusions}

We have shown that a single point mutation in a regulatory domain of the central regulator Xyr1 has tremendous effects on expression behaviour of MIHCs in an industrially used strain of $T$. reesei. We believe that this finding is a very promising starting point for directed strain developments by e.g. transcription factor engineering. Supporting results from $A$. niger suggest that the observed phenomenon is not limited to Trichoderma. Therefore, we recommend manipulations of the regulatory domain of this group of Gal4-like transcription factors as a strategy for inducer-independent expression of MIHCs.

\section{Methods}

\section{Fungal strains}

The following $T$. reesei strains were used throughout this study: the wild-type strain QM6a (ATCC 13631), Rut-C30, which was described as a high yielding cellulase mutant of QM6a (ATCC 56765) [14], Iogen-M4, which is a spontaneous mutant of Rut-C30 [30], Iogen-M8, which is a strain obtained by UV mutation from Iogen-M4, Iogen-M4X, which is a derivate of Iogen-M4 bearing an introduced point mutation (A824V) in Xyr1, and Iogen-M8X, which is a derivate of Iogen-M8 bearing a reconstituted wild-type xyr1. All strains were maintained on malt extract agar or potato-dextrose-agar.

\section{UV mutagenesis and screening}

In order to obtain Iogen-M8, conidia of Iogen-M4 from a single potato dextrose plate were suspended in $10 \mathrm{ml}$ of sterile distilled water and filtered through glass wool to remove any mycelia. The conidia were diluted in water to a concentration of $10^{5}$ per $\mathrm{mL}$ and irradiated in a thin film with a germicidal lamp at a distance of $7 \mathrm{~cm}$. Irradiation for 90-120 seconds was generally sufficient to give 1 - 10\% survival. Diluted suspensions were plated onto selective media containing acid swollen cellulose as the primary carbon source and $4 \mathrm{~g} \mathrm{~L}^{-1}$ of the glucose anti-metabolite 2-deoxyglucose. Iogen-M8 was selected for its ability to grow and produce large clearing zones on this medium, indicative of hyperproduction of cellulose-degrading enzymes.

\section{Growth conditions}

For carbon source replacement experiments, mycelia were pre-cultured in $1 \mathrm{~L}$ Erlenmeyer flasks on a rotary shaker $(180 \mathrm{rpm})$ at $30^{\circ} \mathrm{C}$ for $18 \mathrm{~h}$ in $300 \mathrm{~mL}$ of MandelsAndreotti (MA) medium [31] containing $105 \mathrm{mM}$ of glycerol as the sole carbon source. A total of $10^{9}$ conidia per liter (final concentration) was used as the inoculum. Pre-grown mycelia were washed, and equal amounts were resuspended in MA media containing D-xylose, D-glucose, and sophorose in concentrations as stated. Mycelia were also grown in MA media without any carbon source (control). Samples were taken directly before the carbon source replacement (after harvesting the mycelia after pregrowth), after 3 hours, and after 6 hours of incubation. Samples were derived from three biological replicates and were pooled before RNA extraction.

Cultivations in a bioreactor were run in a $14 \mathrm{~L}$ pilot scale fermentation vessel (Model MF114 New Brunswick Scientific Co.) set up with $10 \mathrm{~L}$ of Initial Pilot Media. Operational parameters were: agitation at $500 \mathrm{rpm}$, air sparging at 8 standard $\mathrm{L} \mathrm{min}^{-1}$, a temperature of $28^{\circ} \mathrm{C}$, and $\mathrm{pH}$ was maintained at 4.0 - 4.5 during batch growth and $\mathrm{pH} 5.0$ during enzyme production. An additional file provides a more detailed bioreactor protocol (see Additional file 5).

Growth on xylan plates was performed using MA medium containing $0.2 \%(\mathrm{w} / \mathrm{v})$ azo-xylan (Megazym, Wicklow, Ireland) at $30^{\circ} \mathrm{C}$.

\section{Determining the relative concentrations of cellulases and hemicellulases}

The relative concentrations of cellulases and hemicellulase mixtures in the culture supernatants produced in bioreactors were determined by ELISA. Supernatants and purified component standards were diluted $1-100 \mu \mathrm{g} \mathrm{mL} \mathrm{m}^{-1}$ in 
phosphate-buffered saline, pH 7.2 (PBS) and incubated overnight at $4{ }^{\circ} \mathrm{C}$ in microtitre plates. The plates were washed with PBS containing 0.1\% Tween 20 (PBS/Tween) and incubated in PBS containing 1\% BSA (PBS/BSA) for $1 \mathrm{~h}$ at room temperature (RT) followed by washing with PBS/Tween. Rabbit polyclonal antisera specific for CBHI, CBHII, EGI, XYNI, XYNII, and BGLI were diluted in PBS/ BSA, added to separate microtitre plates and incubated for $2 \mathrm{~h}$ at RT. Plates were washed and incubated with a goat anti-rabbit antibody coupled to horseradish peroxidase for $1 \mathrm{~h}$ at RT. After washing, tetramethylbenzidine was added and incubated for $1 \mathrm{~h}$ at RT. The absorbance at $660 \mathrm{~nm}$ was measured and converted into protein concentration using the standards. The relative concentration refers to the total protein concentration of the culture supernatants.

\section{Isolation of chromosomal DNA and genome sequencing}

After growth in $75 \mathrm{~mL}$ of MA medium containing $50 \mathrm{mM}$ glucose at $28^{\circ} \mathrm{C}$ for 4 days, cultures were filtered through sterile glass fibre filters and frozen in liquid nitrogen. Biomass was ground to fine powder and resuspended in $30 \mathrm{~mL}$ of lysis buffer (20 mM EDTA, $10 \mathrm{mM}$ Tris- $\mathrm{HCl}$, $\mathrm{pH} 7.9,1 \%$ Triton $\times-100,500 \mathrm{mM}$ guanidine- $\mathrm{HCl}, 200 \mathrm{mM}$ $\mathrm{NaCl}, 0.76 \mathrm{mg} \mathrm{mL}^{-1}$ Driselase ${ }^{\circledR} 0.4 \mathrm{mg} \mathrm{mL}^{-1}$ T. harzianum beta-glucanase, and $0.8 \mu \mathrm{g} \mathrm{mL}^{-1}$ T. viride chitinase C). After treatment with RNase A and RNase T1 at $20 \mu \mathrm{g} \mathrm{m}^{-1}$ and $100 \mathrm{U} \mathrm{mL}^{-1}$ final concentrations $\left(50^{\circ} \mathrm{C}, 1 \mathrm{~h}\right)$, Proteinase $\mathrm{K}$ was added to a concentration of $0.8 \mathrm{mg} \mathrm{mL}^{-1}\left(50^{\circ} \mathrm{C}, 1 \mathrm{~h}\right)$. Following centrifugation $(20 \mathrm{~min}$ at $12,000 \times g)$, the clarified lysate was used to isolate chromosomal DNA using the Qiagen ${ }^{\circledR}$ Genomic-tip 500/G genomic DNA isolation kit (Qiagen Inc.-Canada, Ontario, CA).

Genomic DNA was sequenced using the Illumina/Solexa GAIIx sequencing technology (as distributed by Montreal Biotech Inc., Quebec, Canada) utilizing two lanes per strain (one lane of single read and one lane of paired-end reads). The raw sequences were assembled directly against publicly available sequence for strain QM6a (http://genome.jgipsf.org/Trire2/Trire2.info.html) using DNAstar Seqman NGen ${ }^{\circledR}$ software (DNASTAR Inc., Wisconsin, USA). After assembly, a single nucleotide polymorphism calling procedure was used to identify a table of high-confidence sequence variants.

\section{Plasmid construction}

A $2.6 \mathrm{~kb}$ fragment comprising the 3'-UTR of T. reesei xyr 1 was amplified from Iogen-M4 gDNA using primers FT161f and FT162r. Primer sequences are provided in Table 1. The product was used as a template for a second PCR using primers FT163f and FT164r to generate flanking sequences used in the subsequent recombination steps. Using the In-Fusion HD Cloning System (Clontech Laboratories Inc., California, USA), the second product was recombined with pNCBgl-NN that had been
Table 1 Oligonucleotides used in this study

\begin{tabular}{|c|c|c|}
\hline Name & Sequence $\left(5^{\prime}-3^{\prime}\right)$ & Usage \\
\hline act1f & TGAGAGCGGTGGTATCCACG & \multirow[t]{14}{*}{$q P C R$} \\
\hline act1r & GGTACCACCAGACATGACAATGTTG & \\
\hline cbh1f & GATGATGACTACGCCAACATGCTG & \\
\hline cbh1r & ACGGCACCGGGTGTGG & \\
\hline $\mathrm{cbh} 2 \mathrm{f}$ & CTATGCCGGACAGTTTGTGGTG & \\
\hline cbh2r & GTCAGGCTCAATAACCAGGAGG & \\
\hline $\operatorname{taq} x y n 2 f$ & GGTCCAACTCGGGCAACTTT & \\
\hline $\operatorname{taq} x y n 2 r$ & CCGAGAAGTTGATGACCTTGTTC & \\
\hline taqxyr1f & CCCATTCGGCGGAGGATCAG & \\
\hline taqxyr1r & CGAATTCTATACAATGGGCACATGGG & \\
\hline sar1fw & TGGATCGTCAACTGGTTCTACGA & \\
\hline sar1rev & GCATGTGTAGCAACGTGGTCTTT & \\
\hline xyn1f & CAGCTATTCGCCTTCCAACAC & \\
\hline xyn1r & CAAAGTTGATGGGAGCAGAAG & \\
\hline FT161f & GGAGGCCACTCAATCGTATGA & \multirow[t]{4}{*}{ xyr1 3'-UTR cloning } \\
\hline FT162r & CGTGCCGCAATCCGGTTGTT & \\
\hline FT163f & $\begin{array}{l}\text { TGGGTGGTGGTATAGTCTTAAGGG } \\
\text { AGGCCACTCAATCGTATGA }\end{array}$ & \\
\hline FT164r & $\begin{array}{l}\text { ACGGCCAGTGAATTCTTAATTAA } \\
\text { CGTGCCGCAATCCGGTTGTT }\end{array}$ & \\
\hline FT165f & GATGTGGCAGCCGGGGAA & \multirow{5}{*}{$\begin{array}{l}\text { xyr1 5'-UTR and coding } \\
\text { sequence cloning }\end{array}$} \\
\hline FT166r & TTAGAGGGCCAGACCGGTTC & \\
\hline FT167f & $\begin{array}{l}\text { ACTCTAGATTAATTAAGATGTGGC } \\
\text { AGCCGGGGAA }\end{array}$ & \\
\hline FT168r & $\begin{array}{l}\text { GCTTTCGCCACGGAGCTTAAGAGG } \\
\text { GCCAGACCGGTTC }\end{array}$ & \\
\hline FT169f & $\begin{array}{l}\text { TGCCTGCAGGTCGACTCTAGATTA } \\
\text { ATTAAGATGTGGCAGCCGGGGAA }\end{array}$ & \\
\hline FT170f & $\begin{array}{l}\text { CGGTCTGGCCCTCTAAAGCTCCGT } \\
\text { GGCGAAAGCCT }\end{array}$ & \multirow[t]{2}{*}{ cbhl 3'-UTR cloning } \\
\hline FT171r & $\begin{array}{l}\text { GACGAATGATGGCGGCCGCTT } \\
\text { TCCAGGCCGCCAGCTATG }\end{array}$ & \\
\hline
\end{tabular}

linearized by NheI digestion to produce the intermediate plasmid, pSC1. pNCBgl-NN contains a $3.2 \mathrm{~kb}$ insert comprising the promoter, coding region, and terminator of the $N$. crassa pyr4 gene isolated from plasmid pFB6 [32] and a polylinker with unique NheI and NotI sites located at the $3^{\prime}$ end of the pyr4 insert.

Separately, $4.1 \mathrm{~kb}$ fragments were amplified from Iogen-M4 and Iogen-M8 gDNA using primers FT165f and FT166r comprising the xyr1 5'-UTR and the wild-type xyr1 or xyr1(A824V) coding sequence, respectively. The resulting products were used as templates for a second PCR using primers FT167f and FT166r. FT167f introduces $X b a \mathrm{I}$ and PacI sites at the 5'-end of the amplified products. These PCR products were used as templates for a third PCR using primers FT168r and 
FT169f, to generate fragments suitable for subsequent recombination steps.

A $586 \mathrm{bp}$ fragment comprising the 3'-UTR of the cbh1 gene was amplified from Iogen-M4 gDNA using primers FT170f and FT171r.

pSC1 was linearized using NheI and NotI and recombined with the $4.1 \mathrm{~kb}$ fragment containing the $x y r 15$ '-UTR and xyr 1 or $x y r 1(\mathrm{~A} 824 \mathrm{~V})$ coding sequence and the $586 \mathrm{bp}$ cbh1 3'-UTR fragment using the In-Fusion HD Cloning System (Clontech). This resulted in pSCxyr1-TV and pSCxyr1A824V-TV used for fungal transformation. Vector maps are provided in an additional file (see Additional file 6).

\section{Protoplast transformation}

The protoplast transformation of $T$. reesei was performed as described in U.S. Patent No. 8,323,931. To obtain Iogen$\mathrm{M} 4 \mathrm{X}$, the plasmid $\mathrm{pSCxyr1}{ }^{\mathrm{A} 824 \mathrm{~V}}-\mathrm{TV}$ was transformed into a uridine auxotroph of Iogen-M4 selecting for uridine prototrophy on modified (MA) medium [31]. Introduction of pSCxyr1-TV into a uridine auxotroph of Iogen-M8, followed by selection for uridine prototrophy, resulted in a mutant strain bearing the wild-type $x y r 1$ namely Iogen-M8X.

\section{RNA-extraction and reverse transcription}

Harvested mycelia were homogenized in $1 \mathrm{~mL}$ of peqGOLD TriFast DNA/RNA/protein purification system reagent (PEQLAB Biotechnologie, Erlangen, Germany) using a FastPrep FP120 BIO101 ThermoSavant cell disrupter (Qbiogene, Carlsbad, US). RNA was isolated according to the manufacturer's instructions, and the concentration was measured using the NanoDrop 1000 (Thermo Scientific, Waltham, US).

Synthesis of cDNA from mRNA was carried out using the RevertAid ${ }^{\mathrm{TM}} \mathrm{H}$ Minus First Strand cDNA Synthesis Kit (Fermentas, St. Leon-Rot, Germany) according to the manufacturer's instructions.

\section{Quantitative PCR analysis}

Quantitative PCRs were performed in a Mastercycler ${ }^{\circledR}$ ep realplex 2.2 system (Eppendorf, Hamburg, Germany). All reactions were performed in triplicate. The amplification mixture (final volume $25 \mu \mathrm{L}$ ) contained $12.5 \mu \mathrm{L} 2 \times \mathrm{iQ}$ SYBR Green Mix (Bio-Rad Laboratories, Hercules, USA), $100 \mathrm{nM}$ forward and reverse primer and $2.5 \mu \mathrm{L}$ cDNA (diluted 1:100). Primer sequences are provided in Table 1. Cycling conditions and control reactions were performed as described previously [33]. Data normalization using sar1 and act as reference genes, and calculations were performed as published previously [33]. The transcript levels in all figures were referred to those from QM6a incubated without carbon source for $3 \mathrm{~h}$; therefore, they can be compared cross-figure wisely.

\section{Additional files}

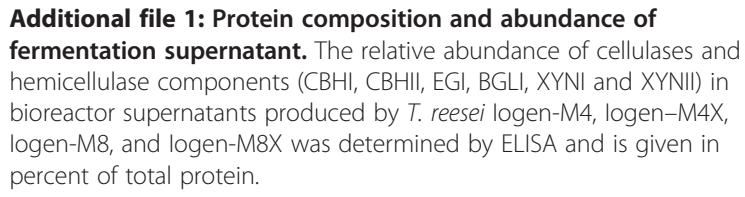

Additional file 2: Gene and aligned protein sequences of wild-type and mutated $x y r 1 / X y r 1$. Intron sequences are shown in italics. Note:

The Trichoderma reesei genome database (http://genome.jgi-psf.org/ Trire2/Trire2.home.html) annotates 3 introns in the xyr 1 mRNA. cDNA sequencing revealed that the middle intron is in fact translated and therefore included in the given sequence. Positions of the mutated site are highlighted in yellow.

Additional file 3: Alignment of the fungal transcription factor regulatory middle homology region (FTFRMH region) of Xyr1 and Cep3p and the consensus. Protein sequences of the FTFRMH region of Xyr1 and Cep3p and the consensus sequence were aligned with COBALT (http://www.ncbi.nlm.nih.gov/tools/cobalt/). Position 824 in Xyr1 is highlighted in red. The helix at M485 to 1475 in Cep3p is highlighted in yellow. Xyr1_dom, FTFRMH region of Xyr1; Cep3p_dom, FTFRMH region of Cep3; Consensus, consensus sequence of the FTFRMH region.

Additional file 4: Graphical representation of 3D structure of dimerized chain A of Cep3p. Protein 3D structure of chain A of Cep3p (PDB: 2VEQA) visualized with Cn3D 4.3. Pictures show a dimer from 3 angles, respectively. The helix at M458 to 1475 is highlighted in yellow in both dimers.

Additional file 5: Detailed protocol for cultivation in a bioreactor.

Additional file 6: Vector maps of pSCxyr1-TV and pSCxyr1A824V-TV. Maps of the vectors pSCxyr1-TV and pSCxyr1A824V-TV used to generate logen M8X and logen-M4X, respectively. Vectors were digested with PaCl prior to transformation of strains logen-M8 and logen-M4.

\section{Abbreviations}

CCR: Carbon catabolite repression; FTFRMH: Fungal transcription factor regulatory middle homology region; MA: Mandels Andreotti; MIHCs: Major, industrially relevant hemicellulases and cellulases; PBS: Phosphate-buffered saline; RT: Room temperature; BSA: Bovine serum albumine; Xyr1: Xylanase regulator 1 .

\section{Competing interests}

LGS, SC, and TW are current or former employees of logen Corporation, which has a commercial interest in the $T$. reesei strains used in this study.

\section{Authors' contributions}

CD carried out transcript analyses and in silico analyses and drafted parts of the manuscript. LGS participated in conception of the study and supervision of experiments. SC constructed strains bearing a mutated Xyr1 and carried out the characterization on the protein level. TW participated in supervision of experiments and revised the manuscript critically. RLM participated in conception of the study and revised the manuscript critically. ARMA participated in conception of the study and prepared the manuscript. All authors read and approved the final manuscript.

\section{Acknowledgements}

This study was supported by logen Energy Corporation and by two grants from the Austrian Science Fund (FWF): [P20192, P24851] given to R.L.M and A.R.M.-A., respectively.

\section{Author details}

${ }^{1}$ Department for Biotechnology and Microbiology, Institute of Chemical Engineering, Vienna University of Technology, Gumpendorfer Str. 1a, A-1060, Wien, Austria. ${ }^{2}$ logen Corp., 310 Hunt Club Rd., Ottawa, ON K1V 1C1, Canada.

Received: 13 December 2012 Accepted: 23 April 2013 Published: 2 May 2013 


\section{References}

1. Kuhls K, Lieckfeldt E, Samuels GJ, Kovacs W, Meyer W, Petrini O, Gams W, Borner T, Kubicek CP: Molecular evidence that the asexual industrial fungus Trichoderma reesei is a clonal derivative of the ascomycete Hypocrea jecorina. Proc Natl Acad Sci U S A 1996, 93:7755-7760.

2. Martinez D, Berka RM, Henrissat B, Saloheimo M, Arvas M, Baker SE, Chapman J, Chertkov O, Coutinho PM, Cullen D, et al: Genome sequencing and analysis of the biomass-degrading fungus Trichoderma reesei (syn. Hypocrea jecorina). Nat Biotechnol 2008, 26:553-560.

3. Teeri T, Salovouri I, Knowles J: The molecular cloning of the major cellulase gene from Trichoderma reesei. Biotechnology 1983, 1:696-699.

4. Törrönen A, Harkki A, Rouvinen J: Three-dimensional structure of endo-1 ,4-beta-xylanase II from Trichoderma reesei: two conformational states in the active site. EMBO J 1994, 13:2493-2501.

5. Vaheri MP, Leisola M, Kaupinnen V: Transgycosylation products of the cellulase system of Trichoderma reesei. Biotechnol Lett 1979, 1:41-46.

6. Stricker $A R$, Mach RL, de Graaff $L H$ : Regulation of transcription of cellulases- and hemicellulases-encoding genes in Aspergillus niger and Hypocrea jecorina (Trichoderma reesei). Appl Microbiol Biotechnol 2008, 78:211-220

7. Mach-Aigner AR, Pucher ME, Mach RL: D-Xylose as a repressor or inducer of xylanase expression in Hypocrea jecorina (Trichoderma reesei). Appl Environ Microbiol 2010, 76:1770-1776.

8. Stricker AR, Grosstessner-Hain K, Würleitner E, Mach RL: Xyr1 (xylanase regulator 1 ) regulates both the hydrolytic enzyme system and D-xylose metabolism in Hypocrea jecorina. Eukaryot Cell 2006, 5:2128-2137.

9. Stricker AR, Steiger MG, Mach RL: Xyr1 receives the lactose induction signal and regulates lactose metabolism in Hypocrea jecorina. FEBS Lett 2007, 581:3915-3920.

10. Mach-Aigner AR, Pucher ME, Steiger MG, Bauer GE, Preis SJ, Mach RL: Transcriptional regulation of $x y r 1$, encoding the main regulator of the xylanolytic and cellulolytic enzyme system in Hypocrea jecorina. Appl Environ Microbiol 2008, 74:6554-6562

11. IImén $M$, Thrane C, Penttilä $M$ : The glucose repressor gene cre1 of Trichoderma: isolation and expression of a full-length and a truncated mutant form. Mol Gen Genet 1996, 251:451-460.

12. Strauss J, Mach RL, Zeilinger S, Hartler G, Stoffler G, Wolschek M, Kubicek CP: Cre1, the carbon catabolite repressor protein from Trichoderma reesei. FEBS Lett 1995, 376:103-107.

13. Mach RL, Strauss J, Zeilinger S, Schindler M, Kubicek CP: Carbon catabolite repression of xylanase I (xyn1) gene expression in Trichoderma reesei. Mol Microbiol 1996, 21:1273-1281.

14. Montenecourt BS, Eveleigh DE: Selective screening methods for the isolation of high yielding cellulase mutants of Trichoderma reesei. In Hydrolysis of cellulose: mechanisms of enzymatic and acid catalysis. Volume 181st edition. Edited by Brown RD, Jurasek L. USA: American Chemical Society; 1979:289-301.

15. Mandels M, Parrish FW, Reese ET: Sophorose as an inducer of cellulase in Trichoderma viride. J Bacteriol 1962, 83:400-408.

16. Margolles-Clark E, Ilmén $M$, Penttilä $M$ : Expression patterns of ten hemicellulase genes of the filamentous fungus Trichoderma reesei on various carbon sources. J Biotechnol 1997, 57:167-179.

17. MacPherson $\mathrm{S}$, Larochelle $\mathrm{M}$, Turcotte $B$ : A fungal family of transcriptional regulators: the zinc cluster proteins. Microbiol Mol Biol Rev 2006, 70:583-604

18. Purvis A, Singleton MR: Insights into kinetochore-DNA interactions from the structure of Cep3Delta. EMBO Rep 2008, 9:56-62

19. Portnoy T, Margeot A, Seidl-Seiboth V, Le Crom S, Ben Chaabane F, Linke R, Seiboth B, Kubicek CP: Differential regulation of the cellulase transcription factors XYR1, ACE2, and ACE1 in Trichoderma reesei strains producing high and low levels of cellulase. Eukaryot Cell 2011, 10:262-271.

20. Hasper AA, Visser J, de Graaff $L H:$ The Aspergillus niger transcriptional activator XInR, which is involved in the degradation of the polysaccharides xylan and cellulose, also regulates D-xylose reductase gene expression. Mol Microbiol 2000, 36:193-200.

21. Tamayo EN, Villanueva A, Hasper AA, de Graaff LH, Ramón D, Orejas M: CreA mediates repression of the regulatory gene $x \ln R$ which controls the production of xylanolytic enzymes in Aspergillus nidulans. Fungal Genet Biol 2008, 45:984-993.

22. Pucher ME, Steiger MG, Mach RL, Mach-Aigner AR: A modified expression of the major hydrolase activator in Hypocrea jecorina (Trichoderma reesei) changes enzymatic catalysis of biopolymer degradation. Catal Today 2011, 167:122-128

23. Würleitner E, Pera L, Wacenovsky C, Cziferszky A, Zeilinger S, Kubicek CP, Mach RL: Transcriptional regulation of xyn2 in Hypocrea jecorina. Eukaryot Cell 2003, 2:150-158

24. Rauscher R, Würleitner E, Wacenovsky C, Aro N, Stricker AR, Zeilinger S, Kubicek CP, Penttilä M, Mach RL: Transcriptional regulation of xyn1, encoding xylanase I, in Hypocrea jecorina. Eukaryot Cell 2006, 5:447-456.

25. Zeilinger S, Mach RL, Kubicek CP: Two adjacent protein binding motifs in the cbh2 (cellobiohydrolase II- encoding) promoter of the fungus Hypocrea jecorina (Trichoderma reesei) cooperate in the induction by cellulose. J Biol Chem 1998, 273:34463-34471.

26. Furukawa T, Shida Y, Kitagami N, Mori K, Kato M, Kobayashi T, Okada H, Ogasawara W, Morikawa Y: Identification of specific binding sites for XYR1, a transcriptional activator of cellulolytic and xylanolytic genes in Trichoderma reesei. Fungal Genet Biol 2009, 46:564-574.

27. Hasper $A A$, Trindade $L M$, van der Veen $D$, van Ooyen AJ, de Graaff $L H$ : Functional analysis of the transcriptional activator XInR from Aspergillus niger. Microbiology 2004, 150:1367-1375.

28. Pace CN, Scholtz JM: A helix propensity scale based on experimental studies of peptides and proteins. Biophys J 1998, 75:422-427.

29. Stone G, Sadowski I: GAL4 is regulated by a glucose-responsive functional domain. EMBO J 1993, 12:1375-1385.

30. Hui J, Lanthier P, White T, McHugh S, Yaguchi M, Roy R, Thibault P: Characterization of cellobiohydrolase I (Cel7A) glycoforms from extracts of Trichoderma reesei using capillary isoelectric focusing and electrospray mass spectrometry. J Chromatogr B 2001, 752:349-368.

31. Mandels M: Applications of cellulases. Biochem Soc Trans 1985, 13:414-416.

32. Buxton FP, Radford A: Cloning of the structural gene for orotidine $5^{\prime}$-phosphate carboxylase of Neurospora crassa by expression in Escherichia coli. Mol Gen Genet 1983, 190:403-405.

33. Steiger MG, Mach RL, Mach-Aigner AR: An accurate normalization strategy for RT-qPCR in Hypocrea jecorina (Trichoderma reesei). J Biotechnol 2010, 145:30-37.

doi:10.1186/1754-6834-6-62

Cite this article as: Derntl et al:: Mutation of the Xylanase regulator 1 causes a glucose blind hydrolase expressing phenotype in industrially used Trichoderma strains. Biotechnology for Biofuels 2013 6:62.

\section{Submit your next manuscript to BioMed Central and take full advantage of:}

- Convenient online submission

- Thorough peer review

- No space constraints or color figure charges

- Immediate publication on acceptance

- Inclusion in PubMed, CAS, Scopus and Google Scholar

- Research which is freely available for redistribution 